\title{
An estimate of Sumudu transforms for Boehmians
}

\author{
Shrideh Khalaf Qasem Al-Omari ${ }^{1}$ and Adem Kılıçman²*
}

\author{
"Correspondence: \\ akilicman@putra.upm.edu.my \\ ${ }^{2}$ Department of Mathematics and \\ Institute of Mathematical Research, \\ Universiti Putra Malaysia (UPM), \\ 43400 UPM, Serdang, Selangor, \\ Malaysia \\ Full list of author information is \\ available at the end of the article
}

\begin{abstract}
The space of Boehmians is constructed using an algebraic approach that utilizes convolution and approximate identities or delta sequences. A proper subspace can be identified with the space of distributions. In this paper, we first construct a suitable Boehmian space on which the Sumudu transform can be defined and the function space $S$ can be embedded. In addition to this, our definition extends the Sumudu transform to more general spaces and the definition remains consistent for $S$ elements. We also discuss the operational properties of the Sumudu transform on Boehmians and finally end with certain theorems for continuity conditions of the extended Sumudu transform and its inverse with respect to $\delta$ - and $\Delta$-convergence. MSC: Primary 54C40; 14E20; secondary 46E25; $20 C 20$
\end{abstract}

Keywords: Sumudu transforms; Boehmian spaces; the space $\mathbb{H}(\mathbb{Y})$; the space $\mathbb{H}\left(\mathbb{Y}^{s}\right)$; distributions

\section{Introduction}

The Sumudu transform of one variable function $f(x)$ is introduced as a new integral transform by Watugala in [1] and is given by

$$
S f(t)(y)=\frac{1}{y} \int_{R_{+}} f(t) \exp \left(\frac{-t}{y}\right) d t, \quad y \in\left(-\tau_{1}, \tau_{2}\right)
$$

over the set of functions

$$
A=\left\{f(t): \exists M, \tau_{1}, \tau_{2}>0,|f(t)|<M e^{\frac{t}{\tau_{j}}}, t \in(-1)^{j} \times(0, \infty)\right\}
$$

where $f(t)$ is a function that can be expressed as a convergent series [2, 3]. The Sumudu transform was applied to solve the ordinary differential equations in control engineering problems; see [3].

The Sumudu transform of the convolution product of $f$ and $u$ is given by

$$
S(f \star u)(y)=y f^{s}(y) u^{s}(y),
$$

where $f^{s}$ and $u^{s}$ are the Sumudu transforms of $f$ and $u$, respectively.

Some of the properties were established by Weerakoon in [4, 5]. In [6], further fundamental properties of this transform were also established by Asiru. Similarly, this transform was applied to a one-dimensional neutron transport equation in [7] by Kadem.

\section{Springer}

(c) 2013 Al-Omari and Kılıçman; licensee Springer. This is an Open Access article distributed under the terms of the Creative Commons Attribution License (http://creativecommons.org/licenses/by/2.0), which permits unrestricted use, distribution, and reproduction in any medium, provided the original work is properly cited. 
In [8], the Sumudu transform was extended to the distributions and some of their properties were also studied. Recently, this transform has been applied to solve the system of differential equations; see Kılıçman et al. in [9].

Note that a very interesting fact about Sumudu transform is that the original function and its Sumudu transform have the same Taylor coefficients except the factor $n$; see Zhang [10]. Similarly, the Sumudu transform sends combinations $C(m, n)$ into permutations $P(m, n)$, and hence it will be useful in the discrete systems.

The following are the general properties of the Sumudu transform which are auxiliary from the substitution method and the properties of integral operators.

(i) If $k_{1}$ and $k_{2}$ are non-negative integers and $S_{1}$ and $S_{2}$ are the corresponding Sumudu transforms of $f_{1}$ and $f_{2}$, respectively, then

$$
S\left(k_{1} f_{1}+k_{2} f_{2}\right)(y)=k_{1} S_{1}(y)+k_{2} S_{2}(y) .
$$

(ii) $S f(k t)(y)=S(k y), k \in R_{+}$.

(iii) $\lim _{t \rightarrow 0} f(t)=\lim _{u \rightarrow 0} S(y)=f(0)$, where $S(y)$ is the Sumudu transform of $f$.

More properties of the Sumudu transforms a long with a some of applications were given in [11] and [12].

\section{Boehmian space}

Boehmians were first constructed as a generalization of regular Mikusinski operators [13]. The minimal structure necessary for the construction of Boehmians consists of the following elements:

(i) a nonempty set $\mathbb{A}$;

(ii) a commutative semigroup $(\mathbb{B}, *)$;

(iii) an operation $\odot: \mathbb{A} \times \mathbb{B} \rightarrow \mathbb{A}$ such that for each $x \in \mathbb{A}$ and $s_{1}, s_{2}, \in \mathbb{B}$, $x \odot\left(s_{1} * s_{2}\right)=\left(x \odot s_{1}\right) \odot s_{2}$

(iv) a collection $\Delta \subset \mathbb{B}^{N}$ such that

(a) If $x, y \in \mathbb{A},\left(s_{n}\right) \in \Delta, x \odot s_{n}=y \odot s_{n}$ for all $n$, then $x=y$;

(b) If $\left(s_{n}\right),\left(t_{n}\right) \in \Delta$, then $\left(s_{n} * t_{n}\right) \in \Delta$.

Elements of $\Delta$ are called delta sequences. Consider

$$
\mathbb{Q}=\left\{\left(x_{n}, s_{n}\right): x_{n} \in \mathbb{A},\left(s_{n}\right) \in \Delta, x_{n} \odot s_{m}=x_{m} \odot s_{n}, \forall m, n \in \mathbf{N}\right\}
$$

Now if $\left(x_{n}, s_{n}\right),\left(y_{n}, t_{n}\right) \in \mathbb{Q}, x_{n} \odot t_{m}=y_{m} \odot s_{n}, \forall m, n \in \mathbf{N}$, then we say $\left(x_{n}, s_{n}\right) \sim\left(y_{n}, t_{n}\right)$. The relation $\sim$ is an equivalence relation in $\mathbb{Q}$. The space of equivalence classes in $\mathbb{Q}$ is denoted by $\beta$. Elements of $\beta$ are called Boehmians.

We note that between $\mathbb{A}$ and $\beta$ there is a canonical embedding expressed as $x \rightarrow \frac{x \odot s_{n}}{s_{n}}$. The operation $\odot$ can also be extended to $\beta \times \mathbb{A}$ by $\frac{x_{n}}{s_{n}} \odot t=\frac{x_{n} \odot t}{s_{n}}$. The relationship between the notion of convergence and the product $\odot$ is given by:

(i) If $f_{n} \rightarrow f$ as $n \rightarrow \infty$ in $\mathbb{A}$ and $\phi \in \mathbb{B}$ is any fixed element, then $f_{n} \odot \phi \rightarrow f \odot \phi$ in $A$ (as $n \rightarrow \infty$ );

(ii) If $f_{n} \rightarrow f$ as $n \rightarrow \infty$ in $\mathbb{A}$ and $\left(\delta_{n}\right) \in \Delta$, then $f_{n} \odot \delta_{n} \rightarrow f$ in $\mathbb{A}$ (as $n \rightarrow \infty$ ).

The operation $\odot$ can be extended to $\beta \times \mathbb{B}$ as follows: If $\left[\frac{f_{n}}{s_{n}}\right] \in \beta$ and $\phi \in \mathbb{B}$, then $\left[\frac{f_{n}}{s_{n}}\right] \odot \phi=$ $\left[\frac{f_{n} \odot \phi}{s_{n}}\right]$. In $\beta$, there are two types of convergence as follows. 
(1) A sequence $\left(h_{n}\right)$ in $\beta$ is said to be $\delta$-convergent to $h$ in $\beta$, denoted by $h_{n} \stackrel{\delta}{\rightarrow} h$, if there exists $\left(s_{n}\right) \in \Delta$ such that $\left(h_{n} \odot s_{n}\right),\left(h \odot s_{n}\right) \in \mathbb{A}, \forall k, n \in \mathbf{N}$, and $\left(h_{n} \odot s_{k}\right) \rightarrow\left(h \odot s_{k}\right)$ as $n \rightarrow \infty$ in $\mathbb{A}$ for every $k \in \mathbf{N}$.

(2) A sequence $\left(h_{n}\right)$ in $\beta$ is said to be $\Delta$-convergent to $h$ in $\beta$, denoted by $h_{n} \stackrel{\Delta}{\rightarrow} h$, if there exists a $\left(s_{n}\right) \in \Delta$ such that $\left(h_{n}-h\right) \odot s_{n} \in \mathbb{A}, \forall n \in \mathbf{N}$, and $\left(h_{n}-h\right) \odot s_{n} \rightarrow 0$ as $n \rightarrow \infty$ in $\mathbb{A}$.

For further discussion, see [14-16].

\section{The Boehmian space $\mathbb{H}(\mathbb{Y})$}

Denote by $\mathbb{S}_{+}(\mathbb{R})$ and $\mathbb{D}_{+}(\mathbb{R})$ the space of all rapidly decreasing functions over $\mathbb{R}_{+}\left(\mathbb{R}_{+}=\right.$ $(0, \infty))$ and the space of all test functions of compact support, respectively. In what follows, we obtain preliminary results required to construct the Boehmian space $\mathbb{H}(\mathbb{Y})$, where $\mathbb{Y}=$ $\left(\mathbb{S}_{+}, \mathbb{D}_{+}, \Delta_{+}\right)$.

\section{Lemma 3.1}

(1) If $u_{1}, u_{2} \in \mathbb{D}_{+}(\mathbb{R})$, then $u_{1} \star u_{2} \in \mathbb{D}_{+}(\mathbb{R})$.

(2) If $f_{1}, f_{2} \in \mathbb{S}_{+}(\mathbb{R})$ and $u_{1} \in \mathbb{D}_{+}(\mathbb{R})$, then $\left(f_{1}+f_{2}\right) \star u_{1}=f_{1} \star u_{1}+f_{2} \star u_{1}$.

(3) $u_{1} \star u_{2}=u_{2} \star u_{1}, \forall u_{1}, u_{2} \in \mathbb{D}_{+}(\mathbb{R})$.

(4) If $f \in S_{+}, u_{1}, u_{2} \in \mathbb{D}_{+}(\mathbb{R})$, then $\left(f \star u_{1}\right) \star u_{2}=f \star\left(u_{1} \star u_{2}\right)$.

Proofs are analogous to those of classical cases and details are omitted.

Definition 3.2 A sequence $\left(s_{n}\right)$ of functions from $\mathbb{D}_{+}(\mathbb{R})$ is said to be in $\Delta_{+}$if and only if

$$
\begin{array}{ll}
\Delta_{+}^{1} & \int_{\mathbf{R}_{+}} s_{n}(x) d x=1 ; \\
\Delta_{+}^{2} & \int_{\mathbf{R}_{+}}\left|s_{n}(x)\right| d x \leq M, \quad M \text { is a positive integer; } \\
\Delta_{+}^{3} & \operatorname{supp} s_{n}(x) \subset\left(0, \varepsilon_{n}\right), \quad \varepsilon_{n} \rightarrow 0 \text { as } n \rightarrow \infty .
\end{array}
$$

This means that $\left(s_{n}\right)$ shrinks to zero as $n \rightarrow \infty$. Each member of $\Delta_{+}$is called a delta sequence or an approximate identity or, sometimes, a summability kernel. Delta sequences, in general, appear in many branches of mathematics, but probably the most important applications are those in the theory of generalized functions. The basic use of delta sequences is the regularization of generalized functions, and further, they can be used to define the convolution product and the product of generalized functions.

Lemma 3.3 If $\left(s_{n}\right),\left(t_{n}\right) \in \Delta_{+}$, then $\operatorname{supp}\left(s_{n} \star t_{n}\right) \subset \operatorname{supp} s_{n}+\operatorname{supp} t_{n}$.

Lemma 3.4 If $u_{1}, u_{2} \in \mathbb{D}_{+}(\mathbb{R})$, then so is $u_{1} \star u_{2}$ and

$$
\int_{\mathbb{R}_{+}}\left|u_{1} \star u_{2}\right| \leq \int_{\mathbb{R}_{+}}\left|u_{1}\right| \cdot \int_{\mathbb{R}_{+}}\left|u_{2}\right|
$$

Theorem 3.5 Let $f_{1}, f_{2} \in \mathbb{S}_{+}(\mathbb{R})$ and $\left(s_{n}\right) \in \Delta_{+}$such that $f_{1} \star s_{n}=f_{2} \star s_{n}, n=1,2,3, \ldots$, then $f_{1}=f_{2}$ in $\mathbb{S}_{+}(\mathbb{R})$. 
Proof We show that $f_{1} \star s_{n}=f_{1}$ in $\mathbb{S}_{+}(\mathbb{R})$. Let $K$ be a compact set containing the supp $s_{n}$ for every $n \in \mathbf{N}$. Using $\Delta_{+}^{1}$, we write

$$
\left|x^{k} D^{m}\left(f_{1} \star s_{n}-f_{1}\right)(x)\right| \leq \int_{K}\left|s_{n}(t)\right|\left|x^{k} D^{m}\left(f_{1}(x-t)-f_{1}(x)\right)\right| d t .
$$

The mapping $t \rightarrow f_{1}^{t}$, where $f_{1}^{t}(x)=f_{1}(x-t)$, is uniformly continuous from $\mathbb{R}_{+} \rightarrow \mathbb{R}_{+}$. From the hypothesis that $\operatorname{supp} s_{n} \rightarrow 0$ as $n \rightarrow \infty$ (by $\Delta_{+}^{3}$ ), we choose $r>0$ such that $\operatorname{supp} s_{n} \subseteq$ $[0, r]$ for large $n$ and $t<r$. This implies

$$
\left|f_{1}(x-t)-f_{1}(x)\right|=\left|f_{1}^{t}-f_{1}\right|<\frac{\varepsilon_{n}}{M}
$$

Hence using $\Delta_{+}^{2}$ and (3.2), (3.1) becomes

$$
\left|x^{k} D^{m}\left(f_{1} \star s_{n}-f_{1}\right)(x)\right|<\varepsilon_{n} \rightarrow 0 \quad \text { as } n \rightarrow \infty .
$$

Thus $f_{1} \star s_{n} \rightarrow f_{1}$ in $\mathbb{S}_{+}(\mathbb{R})$. Similarly, we show that $f_{2} \star s_{n} \rightarrow f_{2}$. This completes the proof of the theorem.

Theorem 3.6 If $\lim _{n \rightarrow \infty} f_{n}=f$ in $\mathbb{S}_{+}(\mathbb{R})$ and $u \in \mathbb{D}_{+}(\mathbb{R})$, then

$$
\lim _{n \rightarrow \infty} f_{n} \star u=f \star u
$$

Proof In view of the hypothesis of the theorem, we write

$$
\left|x^{k} D^{m}\left(f_{n} \star u-f \star u\right)(x)\right|=\left|x^{k}\left(D^{m}\left(f_{n}-f\right) \star u\right)(x)\right| .
$$

The last equation follows from the fact that [17]

$$
D^{m} f \star u=D^{m} f \star u=f \star D^{m} u
$$

Hence, for each $u \in \mathbb{D}_{+}(\mathbb{R})$, we have

$$
\begin{aligned}
\left|x^{k} D^{m}\left(f_{n} \star u-f \star u\right)(x)\right| & \leq \int_{K} x^{k}\left|D^{m}\left(f_{n}-f\right)(x-t)\right||u(t)| d t \\
& \leq M \gamma_{k}\left(f_{n}-f\right) \quad \text { for some constant } M \\
& \rightarrow 0 \quad \text { as } n \rightarrow \infty
\end{aligned}
$$

The proof of the theorem is completed.

Theorem 3.7 If $\lim _{n \rightarrow \infty} f_{n}=f$ in $\mathbb{S}_{+}(\mathbb{R})$ and $\left(s_{n}\right) \in \Delta_{+}$, then $\lim _{n \rightarrow \infty} f_{n} \star s_{n}=f$.

Proof In view of the analysis employed for Theorem 3.5, we get

$$
\lim _{n \rightarrow \infty} f_{n} \star s_{n}=f_{n} \rightarrow f \quad \text { as } n \rightarrow \infty .
$$


Hence

$$
\lim _{n \rightarrow \infty} f_{n} \star s_{n}=f \quad \text { as } n \rightarrow \infty
$$

This completes the proof. The Boehmian space $\mathbb{H}(\mathbb{Y})$ is therefore constructed.

The canonical embedding between $\mathbb{S}_{+}(\mathbb{R})$ and $\mathbb{H}(\mathbb{Y})$ is expressed as $x \rightarrow\left[\frac{x * s_{n}}{s_{n}}\right]$. The extension of $\star$ to $\mathbb{H}(\mathbb{Y}) \times \mathbb{S}_{+}$is given by $\left[\frac{x_{n}}{s_{n}}\right] \star t=\left[\frac{x_{n} \star t}{s_{n}}\right]$. Convergence in $\mathbb{H}(\mathbb{Y})$ is defined in a natural way:

$\delta$-convergence: $A$ sequence $\left(h_{n}\right)$ in $\mathbb{H}(\mathbb{Y})$ is said to be $\delta$-convergent to $h$ in $\mathbb{H}(\mathbb{Y})$, denoted by $h_{n} \stackrel{\delta}{\rightarrow} h$, if there exists a delta sequence $\left(s_{n}\right)$ such that $\left(h_{n} \star s_{n}\right),\left(h \star s_{n}\right) \in \mathbb{S}_{+}(\mathbb{R}), \forall k, n \in \mathbf{N}$, and $\left(h_{n} \star s_{k}\right) \rightarrow\left(h \star s_{k}\right)$ as $n \rightarrow \infty$ in $\mathbb{S}_{+}(\mathbb{R})$ for every $k \in \mathbf{N}$.

$\Delta_{+}$-convergence: $A$ sequence $\left(h_{n}\right)$ in $\mathbb{H}(\mathbb{Y})$ is said to be $\Delta_{+}$-convergent to $h$ in $\mathbb{H}(\mathbb{Y})$, denoted by $h_{n} \stackrel{\Delta}{\rightarrow} h$, if there exists a $\left(s_{n}\right) \in \Delta_{+}$such that $\left(h_{n}-h\right) \star s_{n} \in \mathbb{S}_{+}(\mathbb{R}), \forall n \in N$, and $\left(h_{n}-h\right) \star s_{n} \rightarrow 0$ as $n \rightarrow \infty$ in $\mathbb{S}_{+}(\mathbb{R})$.

Theorem 3.8 The mapping $f \rightarrow\left[\frac{f \star s_{n}}{s_{n}}\right]$ is a continuous embedding of $\mathbb{S}_{+}(\mathbb{R})$ into $\mathbb{H}(\mathbb{Y})$.

Proof The mapping is one-to-one. For detailed proof, let $\left[\frac{f_{1} \star s_{n}}{s_{n}}\right]=\left[\frac{f_{2} \star t_{n}}{t_{n}}\right]$, then $\left(f_{1} \star s_{n}\right) \star t_{m}=$ $\left(f_{2} \star t_{m}\right) \star s_{n}$. Then since $\left(s_{n}\right),\left(t_{n}\right) \in \Delta_{+}, f_{1} \star\left(s_{m} \star t_{n}\right)=f_{2} \star\left(t_{n} \star s_{m}\right)=f_{2} \star\left(s_{m} \star t_{n}\right)$. Using Theorem 3.5, we get $f_{1}=f_{2}$. To show the mapping is continuous, let $f_{n} \rightarrow 0$ as $n \rightarrow \infty$ in $\mathbb{S}_{+}(\mathbb{R})$. Then we show that

$$
\left[\frac{f_{n} \star s_{m}}{s_{m}}\right] \stackrel{\delta}{\rightarrow} 0 \quad \text { as } n \rightarrow \infty
$$

From Theorem 3.5, $\left[\frac{f_{n} \star s_{m}}{s_{m}}\right] \star s_{m}=f_{n} \star s_{m} \rightarrow 0$ as $n \rightarrow \infty$. This completes the proof of the theorem.

Theorem 3.9 Let $f \in \mathbb{S}_{+}(\mathbb{R})$ and $u \in \mathbb{D}_{+}(\mathbb{R})$, then

$$
S(f \star u)(y)=y f^{s}(y) u^{s}(y) .
$$

\section{The Boehmian space $\mathbb{H}\left(\mathbb{Y}^{s}\right)$}

We describe another Boehmian space as follows. Let $\mathbb{S}_{+}(\mathbb{R})$ be the space of rapidly decreasing functions [17]. Define

$$
\mathbb{D}_{+}^{s}(\mathbb{R})=\left\{u^{s}: \text { for all } u \in \mathbb{D}_{+}(\mathbb{R})\right\},
$$

where $u^{s}$ denotes the Sumudu transform of $u$. We also define $f \bullet u^{s}$ by

$$
\left(f \bullet u^{s}\right)(y)=y f(y) u^{s}(y)
$$

Lemma 4.1 Let $f \in \mathbb{S}_{+}(\mathbb{R})$ and $u^{s} \in \mathbb{D}_{+}^{s}(\mathbb{R})$, then $f \bullet u^{s} \in \mathbb{S}_{+}(\mathbb{R})$. 
Proof If $f \in \mathbb{S}_{+}(\mathbb{R})$ and $u^{s} \in \mathbb{D}_{+}^{s}(\mathbb{R})$, then using the topology of $\mathbb{S}_{+}(\mathbb{R})$ and Leibnitz' theorem, we get

$$
\begin{aligned}
\left|x^{k} D_{x}^{m}\left(f \bullet u^{s}\right)(x)\right| & \leq\left|x^{k} \sum_{j=1}^{m} D^{m-j}(x f(x)) D^{j} u^{s}(x)\right| \\
& \leq \sum_{j=1}^{m}\left|x^{k} D^{m-j}(x f(x))\right|\left|D^{j} u^{s}(x)\right| \\
& =\sum_{j=1}^{m}\left|x^{k} D^{m-j} f_{1}(x)\right|\left|\int_{K} u(t) D_{x}^{j} \frac{e^{-\frac{t}{x}}}{x} d t\right|,
\end{aligned}
$$

where $f_{1}(x)=x f(x) \in \mathbb{S}_{+}(\mathbb{R})$ and $K$ is a compact subset containing the supp $u(t)$. Hence

$$
\left|x^{k} D_{x}^{m}\left(f \bullet u^{s}\right)(x)\right| \leq M \gamma_{k, m-j}\left(f_{1}\right)<\infty
$$

for some positive constant $M$. This completes the proof of the lemma.

Lemma 4.2 The mapping

$$
\begin{aligned}
& \mathbb{S}_{+} \times \mathbb{D}_{+}^{s} \rightarrow \mathbb{S}_{+}, \\
& \left(f, u^{s}\right) \rightarrow f \bullet u^{s}
\end{aligned}
$$

satisfies the following properties:

(1) If $u_{1}^{s}, u_{2}^{s} \in \mathbb{D}_{+}^{s}(\mathbb{R})$, then $u_{1}^{s} \bullet u_{2}^{s} \in \mathbb{D}_{+}^{s}(\mathbb{R})$.

(2) If $f_{1}, f_{2} \in \mathbb{S}_{+}(\mathbb{R}), u^{s} \in \mathbb{D}_{+}^{s}(\mathbb{R})$, then $\left(f_{1}+f_{2}\right) \bullet u^{s}=f_{1} \bullet u^{s}+f_{2} \bullet u^{s}$.

(3) For $u_{1}^{s}, u_{2}^{s} \in \mathbb{D}_{+}^{s}(\mathbb{R}), u_{1}^{s} \bullet u_{2}^{s}=u_{2}^{s} \bullet u_{1}^{s}$.

(4) For $f \in \mathbb{S}_{+}(\mathbb{R}), u_{1}^{s}, u_{2}^{s} \in \mathbb{D}_{+}^{s}(\mathbb{R})$, then $\left(f \bullet u_{1}^{s}\right) \bullet u_{2}^{s}=f \bullet\left(u_{1}^{s} \bullet u_{2}^{s}\right)$.

Proof The proof of the above lemma is straightforward. Detailed proof is as follows.

Proof of $(1)$. Let $u_{1}, u_{2} \in \mathbb{D}_{+}(\mathbb{R})$, then $u_{1} \star u_{2} \in \mathbb{D}_{+}(\mathbb{R})$. Hence $\left(u_{1} \star u_{2}\right)^{s} \in \mathbb{D}_{+}^{s}(\mathbb{R})$ by $(4.1)$.

Theorem 3.9 implies $u_{1}^{s} \bullet u_{2}^{s} \in \mathbb{D}_{+}^{s}(\mathbb{R})$.

Proof of (2) is obvious.

Proof of (3). We have

$$
\begin{aligned}
\left(u_{1}^{s} \bullet u_{2}^{s}\right)(x) & =x u_{1}^{s}(x) u_{2}^{s}(x) \\
& =x u_{2}^{s}(x) u_{1}^{s}(x) \\
& =\left(u_{2}^{s} \bullet u_{1}^{s}\right)(x) .
\end{aligned}
$$

Hence $u_{1}^{s} \bullet u_{2}^{s}=u_{2}^{s} \bullet u_{1}^{s}$.

Proof of (4). Let $f \in \mathbb{S}_{+}(\mathbb{R}), u_{1}^{s}, u_{2}^{s} \in \mathbb{D}_{+}^{s}(\mathbb{R})$, then

$$
\begin{aligned}
\left(\left(f \bullet u_{1}^{s}\right) \bullet u_{2}^{s}\right)(x) & =x\left(f \bullet u_{1}^{s}\right)(x) u_{2}^{s} \\
& =x x f(x) u_{1}^{s}(x) u_{2}^{s}(x) \\
& =x f(x) x u_{1}^{s}(x) u_{2}^{s}(x)
\end{aligned}
$$




$$
\begin{aligned}
& =x f(x)\left(u_{1}^{s} \bullet u_{2}^{s}\right)(x) \\
& =f \bullet\left(u_{1}^{s} \bullet u_{2}^{s}\right)(x),
\end{aligned}
$$

that is,

$$
\left(f \bullet u_{1}^{s}\right) \bullet u_{2}^{s}=f \bullet\left(u_{1}^{s} \bullet u_{2}^{s}\right)
$$

This completes the proof of the theorem.

Denote by $\Delta_{+}^{s}$ the set of all Sumudu transforms of delta sequences from $\Delta_{+}$. That is,

$$
\Delta_{+}^{s}=\left\{\left(s_{n}^{s}\right):\left(s_{n}\right) \in \Delta_{+}, \forall n \in \mathbf{N}\right\} .
$$

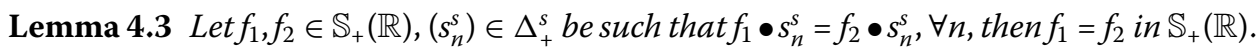

Proof Let $f_{1}, f_{2} \in \mathbb{S}_{+}(\mathbb{R})$ and $\left(s_{n}^{s}\right) \in \Delta_{+}^{s}$. Since $f_{1} \bullet s_{n}^{s}=f_{2} \bullet s_{n}^{s}$, (4.2) implies $x f_{1}(x) s_{n}^{s}(x)=$ $x f_{2}(x) s_{n}^{s}(x)$. Hence $f_{1}(x)=f_{2}(x)$ for all $x$. The proof is completed.

Lemma 4.4 For each $\left(s_{n}\right),\left(t_{n}\right) \in \Delta_{+},\left(s_{n}^{s} \bullet t_{n}^{s}\right) \in \Delta_{+}^{s}$.

Proof Since $\left(s_{n}\right),\left(t_{n}\right) \in \Delta_{+}, s_{n} \star t_{n} \in \Delta_{+}$for all $n$. Hence, from Theorem 3.9, we get $S\left(s_{n} \star\right.$ $\left.t_{n}\right)(x)=x s_{n}^{s}(x) t_{n}^{s}(x)=s_{n}^{s} \bullet t_{n}^{s} \in \Delta_{+}^{s}$ for every $n$. This completes the proof of the lemma.

By aid of Lemma 4.3. and Lemma 4.4, $\Delta_{+}^{s}$ can be regarded as a delta sequence.

Lemma 4.5 Let $f_{n} \rightarrow f$ in $\mathbb{S}_{+}(\mathbb{R}), u^{s} \in \mathbb{D}_{+}^{s}(\mathbb{R})$, then $f_{n} \bullet u^{s} \rightarrow f \bullet u^{s}$ in $\mathbb{S}_{+}(\mathbb{R})$.

Proof It is clear that $u^{s}$ is bounded in $\mathbb{D}_{+}^{s}(\mathbb{R})$. Further,

$$
\begin{aligned}
\left(f_{n} \bullet u^{s}\right)(x) & \rightarrow x f(x) u^{s}(x) \\
& \rightarrow\left(f_{n} \bullet u^{s}\right)(x) .
\end{aligned}
$$

Hence $\left(f_{n} \bullet u^{s}\right) \rightarrow f \bullet u^{s}$.

Lemma 4.6 $\operatorname{Let}_{n} \rightarrow f$ in $\mathbb{S}_{+}(\mathbb{R}),\left(s_{n}^{s}\right) \in \Delta_{+}^{s}$, then $f_{n} \bullet s_{n}^{s} \rightarrow f$ in $\mathbb{S}_{+}(\mathbb{R})$.

Proof Let $\left(s_{n}\right) \in \Delta_{+}$, then $s_{n}^{s}(x) \rightarrow \frac{1}{x}$ uniformly on compact subsets of $\mathbb{R}_{+}$. Hence

$$
\begin{aligned}
\left|x^{k} D_{x}^{m}\left(f_{n} \bullet s_{n}^{s}-f\right)(x)\right| & =\left|x^{k} D_{x}^{m}\left(x f_{n}(x) s_{n}^{s}(x)-f(x)\right)\right| \\
& \rightarrow\left|x^{k} D_{x}^{m}\left(f_{n}-f\right)(x)\right|
\end{aligned}
$$

as $n \rightarrow \infty$. Thus $\left|x^{k} D_{x}^{m}\left(f_{n} \bullet s_{n}^{s}-f\right)(x)\right| \rightarrow 0$ as $n \rightarrow \infty$. This yields $f_{n} \bullet s_{n}^{s} \rightarrow f$ in the topology of $\mathbb{S}_{+}(\mathbb{R})$. The proof is therefore completed. The space $\mathbb{H}\left(\mathbb{Y}^{s}\right)$ can be regarded as a Boehmian space, where $\mathbb{Y}^{s}=\left(\mathbb{S}_{+}, \mathbb{D}_{+}^{s}, \Delta_{+}^{s}\right)$. 
Lemma 4.7 The mapping

$$
f \rightarrow\left[\frac{f \bullet s_{n}^{s}}{s_{n}^{s}}\right]
$$

is a continuous embedding of $\mathbb{S}_{+}(\mathbb{R})$ into $\mathbb{H}\left(\mathbb{Y}^{s}\right)$.

Proof For $f \in \mathbb{S}_{+}(\mathbb{R}), s_{n}^{s} \in \Delta_{+}^{s}, \frac{f \bullet s_{n}^{s}}{s_{n}^{s}}$ is a quotient of sequences in the sense that $\left(f \bullet s_{n}^{s}\right) \bullet s_{m}^{s}=$ $f \bullet\left(s_{m}^{s} \bullet s_{n}^{s}\right)$. We show that the map (4.4) is one-to-one. Let $\left[\frac{f_{1} \bullet s_{n}^{s}}{s_{n}^{s}}\right]=\left[\frac{f_{2} \bullet \bullet_{n}^{s}}{t_{n}^{s}}\right]$, then $\left(f_{1} \bullet s_{n}^{s}\right) \bullet t_{m}^{s}=$ $\left(f_{2} \bullet t_{m}^{s}\right) \bullet s_{n}^{s}, m, n \in \mathbf{N}$. Using Lemma 4.2 and Lemma 4.3, we conclude $f_{1}=f_{2}$.

To establish the continuity of (4.4), let $f_{n} \rightarrow 0$ as $n \rightarrow \infty$ in $\mathbb{S}_{+}(\mathbb{R})$. Then $f_{n} \bullet s_{n}^{s} \rightarrow 0$ as $n \rightarrow \infty$ by Lemma 4.6 , and hence

$$
\left[\frac{f_{n} \bullet s_{n}^{s}}{s_{n}^{s}}\right] \rightarrow 0
$$

as $n \rightarrow \infty$ in $\mathbb{H}\left(\mathbb{Y}^{s}\right)$. This completes the proof of the lemma.

\section{The Sumudu transform of Boehmians}

Let $\beta=\left[\frac{f_{n}}{s_{n}}\right] \in \mathbb{H}(\mathbb{Y})$, then we define the Sumudu transform of $\beta$ by the relation

$$
\beta_{1}^{s}=\left[\frac{f_{n}^{s}}{s_{n}^{s}}\right] \quad \text { in } \mathbb{H}\left(\mathbb{Y}^{s}\right) .
$$

Theorem 5.1 $\beta_{1}^{s}: \mathbb{H}(\mathbb{Y}) \rightarrow \mathbb{H}\left(\mathbb{Y}^{s}\right)$ is well defined.

Proof Let $\beta_{1}=\beta_{2} \in \mathbb{H}(\mathbb{Y})$, where $\beta_{1}=\left[\frac{f_{n}}{s_{n}}\right], \beta_{2}=\left[\frac{g_{n}}{t_{n}}\right]$. Then the concept of quotients yields $f_{n} \star t_{m}=g_{m} \star s_{n}$. Employing Theorem 3.9, we get $x f_{n}^{s}(x) t_{m}^{s}(x)=x g_{m}^{s}(x) s_{n}^{s}(x)$, i.e., $f_{n}^{s} \bullet t_{m}^{s}=$ $g_{m}^{s} \bullet s_{n}^{s}$. Equivalently, $\frac{f_{n}^{s}}{s_{n}^{s}} \sim \frac{g_{n}^{s}}{t_{n}^{s}}$. Thus $\beta_{1}^{s}=\beta_{2}^{s}$. This completes the proof of the theorem.

Theorem 5.2 $\beta^{s}: \mathbb{H}(\mathbb{Y}) \rightarrow \mathbb{H}\left(\mathbb{Y}^{s}\right)$ is continuous with respect to $\delta$-convergence.

Proof Let $\beta_{n} \rightarrow 0$ in $\mathbb{H}(\mathbb{Y})$, then by [14], $\beta_{n}=\left[\frac{f_{n, k}}{s_{k}}\right]$ and $f_{n, k} \rightarrow 0$ as $n \rightarrow \infty$ in $\mathbb{S}_{+}(\mathbb{R})$. Applying the Sumudu transform to both sides yields $f_{n, k}^{s} \rightarrow 0$ as $n \rightarrow \infty$. Hence

$$
\beta_{n}^{s}=\left[\frac{f_{n, k}^{s}}{s_{k}^{s}}\right] \rightarrow 0
$$

as $n \rightarrow \infty$ in $\mathbb{H}\left(\mathbb{Y}^{s}\right)$. This proves the theorem.

Theorem 5.3 $\beta^{s}: \mathbb{H}(\mathbb{Y}) \rightarrow \mathbb{H}\left(\mathbb{Y}^{s}\right)$ is a one-to-one mapping.

Proof Assume $\beta_{1}^{s}=\left[\frac{f_{n}^{s}}{s_{n}^{s}}\right]=\left[\frac{g_{n}^{s}}{t_{n}^{s}}\right]=\beta_{2}^{s}$, then $f_{n}^{s} \bullet t_{m}^{s}=g_{m}^{s} \bullet s_{n}^{s}$. Hence

$$
\left(f_{n} \star t_{m}\right)^{s}=\left(g_{m} \star s_{n}\right)^{s} .
$$

Since the Sumudu transform is one-to-one, we get $f_{n} \star t_{m}=g_{m} \star s_{n}$. Thus

$$
\frac{f_{n}}{s_{n}} \sim \frac{g_{n}}{t_{n}}
$$


Hence

$$
\left[\frac{f_{n}}{s_{n}}\right]=\beta_{1}=\left[\frac{g_{n}}{t_{n}}\right]=\beta_{2} \text {. }
$$

This completes the proof of the theorem.

Theorem 5.4 Let $\beta_{1}, \beta_{2} \in \mathbb{H}(\mathbb{Y})$, then

(1) $\left(\beta_{1}+\beta_{2}\right)^{s}=\beta_{1}^{s}+\beta_{2}^{s}$;

(2) $(k \beta)^{s}=k \beta^{s}, \lambda \in \mathbb{C}$.

Proof is immediate from the definitions.

Theorem $5.5 \beta^{s}: \mathbb{H}(\mathbb{Y}) \rightarrow \mathbb{H}\left(\mathbb{Y}^{s}\right)$ is continuous with respect to $\Delta_{+}$-convergence.

Proof Let $\beta_{n} \stackrel{\Delta}{\rightarrow} \beta$ in $\mathbb{H}(\mathbb{Y})$ as $n \rightarrow \infty$. Then there exist $f_{n} \in \mathbb{S}_{+}(\mathbb{R})$ and $\left(s_{n}\right) \in \Delta_{+}$such that $\left(\beta_{n}-\beta\right) \star s_{n}=\left[\frac{f_{n} \star s_{k}}{s_{k}}\right]$ and $f_{n} \rightarrow 0$ as $n \rightarrow \infty$. Employing Eq. (5.1), we get

$$
S\left(\left(\beta_{n}-\beta\right) \star S_{n}\right)=\left[\frac{S\left(f_{n} \star s_{k}\right)}{s_{k}^{s}}\right] .
$$

Hence, we have $S\left(\left(\beta_{n}-\beta\right) \star s_{n}\right)=\left[\frac{y f_{n}^{s} s_{k}^{s}}{s_{k}^{s}}\right] \rightarrow 0$ as $n \rightarrow \infty$ in $\mathbb{H}\left(\mathbb{Y}^{s}\right)$. Therefore

$$
\begin{aligned}
S\left(\left(\beta_{n}-\beta\right) \star s_{n}\right) & =y\left(\beta_{n}^{s}-\beta^{s}\right) s_{n}^{s} \\
& \rightarrow 0 \quad \text { as } n \rightarrow \infty
\end{aligned}
$$

Hence, $\beta_{n}^{s} \stackrel{\Delta}{\rightarrow} \beta^{s}$ as $n \rightarrow \infty$.

Theorem $5.6 \beta^{s}: \mathbb{H}(\mathbb{Y}) \rightarrow \mathbb{H}\left(\mathbb{Y}^{s}\right)$ is onto.

Proof Let $\left[\frac{f_{n}^{s}}{s_{n}^{s}}\right] \in \mathbb{H}\left(\mathbb{Y}^{s}\right)$ be arbitrary, then $f_{n}^{s} \bullet s_{m}^{s}=f_{m}^{s} \bullet s_{n}^{s}$ for every $m, n \in \mathbf{N}$. Then $f_{n} \star s_{m}=$ $f_{m} \star s_{n}$. That is, $\frac{f_{n}}{s_{n}}$ is the corresponding quotient of sequences of $\frac{f_{n}^{s}}{s_{n}^{s}}$. Thus $\left[\frac{f_{n}}{s_{n}}\right] \in \mathbb{H}(\mathbb{Y})$ is such that $S\left[\frac{f_{n}}{s_{n}}\right]=\left[\frac{f_{n}^{s}}{s_{n}^{s}}\right]$ in $\mathbb{H}\left(\mathbb{Y}^{s}\right)$. This completes the proof of the lemma.

Let $\beta^{s}=\left[\frac{f_{n}^{s}}{s_{n}^{s}}\right] \in \mathbb{H}\left(\mathbb{Y}^{s}\right)$, then we define the inverse Sumudu transform of $\beta^{s}$ by

$$
\beta^{s^{-1}}=\left[\frac{f_{n}}{s_{n}}\right]
$$

in the space $\mathbb{H}(\mathbb{Y})$.

Theorem 5.7 Let $\left[\frac{f_{n}^{s}}{s_{n}^{s}}\right] \in \mathbb{H}\left(\mathbb{Y}^{s}\right)$ and $u \in \mathbb{D}_{+}(\mathbb{R}), u^{s} \in \mathbb{D}_{+}^{s}(\mathbb{R})$

$$
\beta\left(\left[\frac{f_{n}}{s_{n}}\right] \star u\right)=\left[\frac{f_{n}^{s}}{s_{n}^{s}}\right] \bullet u \text { and } \beta^{s^{-1}}\left(\left[\frac{f_{n}^{s}}{s_{n}^{s}}\right] \bullet u^{s}\right)=\left[\frac{f_{n}}{s_{n}}\right] \star u \text {. }
$$

Proof is immediate from the definitions. 


\section{Competing interests}

The authors declare that they have no competing interests.

\section{Authors' contributions}

Both of the authors contributed equally to the manuscript and read and approved the final draft.

\section{Author details}

${ }^{1}$ Department of Applied Sciences, Faculty of Engineering Technology, Al-Balqa Applied University, Amman, 11134 , Jordan. ${ }^{2}$ Department of Mathematics and Institute of Mathematical Research, Universiti Putra Malaysia (UPM), 43400 UPM, Serdang, Selangor, Malaysia.

\section{Acknowledgements}

Dedicated to Professor Hari M Srivastava.

The authors express their sincere thanks to the referee(s) for the careful and detailed reading of the manuscript and very helpful suggestions that improved the manuscript substantially.

Received: 14 January 2013 Accepted: 5 March 2013 Published: 26 March 2013

\section{References}

1. Watugala, GK: Sumudu transform: a new integral transform to solve differential equations and control engineering problems. Int. J. Math. Educ. Sci. Technol. 24(1), 35-43 (1993)

2. Belgacem, FBM, Karaballi, AA, Kalla, LS: Analytical investigations of the Sumudu transform and applications to integral production equations. Math. Probl. Eng. 3, 103-118 (2003)

3. Weerakoon, S: Application of Sumudu transform to partial differential equations. Int. J. Math. Educ. Sci. Technol. 25, 277-283 (1994)

4. Watugala, GK: Sumudu transform new integral transform to solve differential equations and control engineering problems. Math. Eng. Ind. 6(4), 319-329 (1998)

5. Watugala, GK: The Sumudu transform for functions of two variables. Math. Eng. Ind. 8(4), 293-302 (2002)

6. Asiru, MA: Further properties of the Sumudu transform and its applications. Int. J. Math. Educ. Sci. Technol. 33(3), 441-449 (2002)

7. Kadem, A: Solving the one-dimensional neutron transport equation using Chebyshev polynomials and the Sumudu transform. An. Univ. Oradea, Fasc. Mat. 12, 153-171 (2005)

8. Eltayeb, H, Kılıçman, A, Fisher, B: A new integral transform and associated distributions. Integral Transforms Spec. Funct. 21(5), 367-379 (2010)

9. Kılıçman, A, Eltayeb, H, Agarwal, PR: On Sumudu transform and system of differential equations. Abstr. Appl. Anal. 2010, Article ID 598702 (2010). doi:10.1155/2010/598702

10. Zhang, J: A Sumudu based algorithm for solving differential equations. Comput. Sci. J. Mold. 15(3(45)), 303-313 (2007)

11. Weerakoon, S: Complex inversion formula for Sumudu transforms. Int. J. Math. Educ. Sci. Technol. 29(4), 618-621 (1998)

12. Kılıçman, A, Eltayeb, H: On the applications of Laplace and Sumudu transforms. J. Franklin Inst. 347(5), 848-862 (2010)

13. Boehme, TK: The support of Mikusinski operators. Trans. Am. Math. Soc. 176, 319-334 (1973)

14. Mikusinski, P: Fourier transform for integrable Boehmians. Rocky Mt. J. Math. 17(3), 577-582 (1987)

15. Mikusinski, P: Tempered Boehmians and ultradistributions. Proc. Am. Math. Soc. 123(3), 813-817 (1995)

16. Roopkumar, R: Mellin transform for Boehmians. Bull. Inst. Math. Acad. Sin. 4(1), 75-96 (2009)

17. Zemanian, AH: Generalized Integral Transformation. Dover, New York (1987). (First published by interscience publishers)

doi:10.1186/1687-1847-2013-77

Cite this article as: Al-Omari and Kılıçman: An estimate of Sumudu transforms for Boehmians. Advances in Difference Equations 2013 2013:77.

\section{Submit your manuscript to a SpringerOpen ${ }^{\circ}$ journal and benefit from:}

- Convenient online submission

Rigorous peer review

- Immediate publication on acceptance

- Open access: articles freely available online

- High visibility within the field

- Retaining the copyright to your article 